\title{
Aplicabilidad del Monitoreo de Emisiones del Arco Eléctrico Para el Control de Calidad en el Proceso MAG-S
}

\author{
(Applicability of Monitoring of Electric Arc Emissions for Quality Control in MAG-S Process)
}

\author{
Eber Huanca Cayo ${ }^{1}$, Sadek Crisóstomo Absi Alfaro \\ ${ }^{1}$ Universidade de Brasília, Departamento de Engenharia Mecânica, Brasília, Distrito Federal, Brasil, \\ eber@unb.brsadek@unb.br
}

\begin{abstract}
Resumen
Garantizar la calidad en soldadura no es una tarea trivial. Para ello diversas inspecciones de control de calidad son realizadas, en detrimento, los costos y tiempos de producción se elevan. Existen diversos sistemas automatizados de soldadura, éstos son auxiliados por sistemas de control basados en el monitoreo de parámetros de soldadura. Sin embargo son reducidos los sistemas automatizados de monitoreo de calidad que en su mayoría son diseñados para el proceso TIG. Durante la soldadura, el arco eléctrico produce emisiones acústicas y electromagnéticas que se manifiestan como sonido y luminosidad. El objetivo del presente trabajo es mostrar que estas emisiones del arco pueden ser utilizados para el monitoreo de la calidad de la soldadura para el proceso MAG-S. Se realizaron múltiples experimentos de soldadura en posición plana donde se indujeron perturbaciones consistentes presencia de grasa y ausencia de gas de protección. En cada experimento se adquirió simultáneamente señales de tensión y corriente así como señales de emisiones del arco eléctrico. A partir de las emisiones acústicas y electromagnéticas en la banda de ultravioleta, se midió la frecuencia de cortocircuitos. A partir de la emisión electromagnética en la banda infrarroja se midió la estabilidad del proceso de soldadura. Los resultados muestran que las emisiones del arco pueden ser utilizadas para el monitoreo y detección de perturbaciones en soldadura y con el entendimiento de las variaciones de cada emisión podría identificarse determinados tipos de perturbaciones.
\end{abstract}

Palabras clave: MAG-S; Emisiones de Arco; Monitoreo de Calidad.

Abstract: Ensuring quality in welding is not a trivial task. For this purpose several quality control checks are performed, at the expense, costs and production times are increased. There are several automated welding systems and they are assisted by control systems based on monitoring of welding parameters. However, automated quality monitoring systems are limited and they are mostly designed for the TIG welding processes. During welding, the arc produces acoustic and electromagnetic emissions that occur as sound and light. The aim of this paper is to show that the emissions of the welding arc can be used to monitoring the quality of welding in MAG-S process. Multiple welding experiments were performed in flat position; in each experiment were induced perturbations by grease on plate and shielding gas absence. Current and voltage signals and arc emission signals were acquired simultaneously. The short circuit frequency was measured from acoustic and electromagnetic emission in the ultraviolet band. The welding stability was measured from infrared emission. The results show that the emissions of welding arc can be used for monitoring and detecting of disturbances in welding and with the understanding of the variations of each disturbance could be possible to identify certain types of disturbances.

Key-words: MAG-S; Arc Emissions; Quality Monitoring.

\section{Introducción}

La soldadura de arco con protección gaseosa en el modo de transferencia por corto-circuito (que en adelante será nombrada como MAG-S) es un proceso de fabricación ampliamente utilizado para la unión de materiales metálicos; presenta como principales ventajas la elevada tasa de transferencia metálica, costos reducidos de mantenimiento, facilidad de automatización así como una considerable estabilidad al soldar en diversas posiciones. Según la aplicación de los productos fabricados que utilizan la soldadura, existen diversos requerimientos

(Recebido em 03/01/2011; Texto final em 29/08/2011). de calidad y para ello son llevados a cabo desde simples inspecciones visuales hasta testes exhaustivos como los ensayos destructivos y no destructivos lo que conlleva al incremento de los tiempos y costos de fabricación. La automatización de los procesos de soldadura ha permitido reducir los tiempos y costos de fabricación pero no sucede lo mismo con los tiempos y costos de inspección y control de calidad que inevitablemente dependen del juicio de inspectores experimentados y calificados. No obstante ello en un esfuerzo por reducir los tiempos de control de calidad, se han desarrollado sistemas automatizados de monitoreo de calidad basado en la medición de algunos parámetros de soldadura tales como la tensión y corriente que junto a técnicas basadas en sistemas de control estadístico han presentado cierto nivel de confiabilidad. Estos sistemas se basan en la estabilidad estadística (estacionariedad) de los parámetros monitoreados donde un cambio súbito sea éste mayor o menor 
a ciertos límites pre establecidos indicará la presencia de alguna inestabilidad anormal que podría ser considerado un defecto o en todo caso una soldadura que esta fuera del padrón establecido previamente. El proceso de soldadura presenta múltiples variables de entrada y de salida que están interrelacionadas sinérgicamente entre una y otra; esto hace que el comportamiento de la transferencia metálica sea de naturaleza estocástica por lo que la estacionariedad de los parámetros monitoreados es una medida de estabilidad de la transferencia metálica.

Parámetros como la tensión y corriente velocidad son responsables por la fusión de las piezas soldadas donde el arco eléctrico aporta calor a la poza de fusión originando oscilaciones en la columna de plasma del arco así como de la misma poza y que como consecuencia, genera emisiones de naturaleza acústica y electromagnética. Es sabido que los soldadores experimentados utilizan las emisiones de arco en forma de una combinación de informaciones acústicas y visuales para monitorear, controlar y mantener la calidad de la soldadura a niveles aceptables. Diversas investigaciones confirmaron que existe una relación entre los parámetros de soldadura y las emisiones del arco eléctrico que permite al soldador tener cierto grado de control sobre el proceso de soldadura [1-4]. El objetivo de este trabajo es mostrar que las emisiones del arco eléctrico además de mostrar una relación con los parámetros de soldadura, pueden ser utilizadas también como parámetros de evaluación de calidad en el proceso MAG-S, pudiendo detectar e identificar determinadas perturbaciones inducidas.

\subsection{Sistemas Automatizados de Monitoreo de Calidad de Soldadura}

Los sistemas automatizados de monitoreo de calidad en su mayoría están basados en la técnica de procesamiento de padrones de imágenes (Signature Image Processing - SIP) [5]. Se trata de una tecnología de análisis de datos eléctricos adquiridos durante la soldadura y que a través de un procesamiento en tiempo real y con el apoyo de algoritmos de evaluación de estacionariedad de datos, indica finalmente el nivel de calidad de la soldadura. Estos sistemas de evaluación de calidad tienen como objetivo mostrar la presencia o no de interferencia en el proceso de soldadura sin desempeñar tareas de control y/o corrección. Diversos autores han desarrollado técnicas y algoritmos de control de transferencia metálica en soldadura [6-9], lo que ha implicado el monitoreo de algunos parámetros que fueron utilizados para cerrar el lazo de control. La fusión de estos sistemas de control y monitoreo ha dado origen a los sistemas de evaluación de calidad y corrección de perturbaciones y que de acuerdo a la complejidad de las operaciones que desempeñan (ver figura 1), estos sistemas pueden clasificarse en tres niveles [10]. En el primer nivel es posible detectar soldaduras defectuosas automáticamente durante la producción a partir del monitoreo de la tensión y/o la corriente de soldadura. En el segundo nivel es posible localizar e identificar los defectos y las posibles causas de la falla en la soldadura (cambios en el proceso de soldadura inducidos por disturbios en la protección de gas, cambios en la velocidad de alimentación de electrodo así como en la geometría de la unión, etc.), a partir del monitoreo de parámetros adicionales como la velocidad de soldadura, velocidad de alimentación de electrodo, etc. En el tercer nivel es posible desempeñar tareas de control sobre los parámetros del proceso para corregir la presencia de perturbaciones, esto se logra a partir de técnicas de procesamiento, identificación y clasificación de datos.

Para el control y corrección de calidad han sido utilizadas diversas técnicas de control así como también monitoreado diversos parámetros de soldadura tales como la tensión y la corriente. El monitoreo de emisiones de arco no ha sido ajeno a estos sistemas. Como se puede observar en la Figura 2, las emisiones del arco manifestadas como sonido y luminosidad, han sido comúnmente utilizadas para detectar la presencia de interferencias. Para la identificación y corrección de interferencias se han utilizado algunas bandas de frecuencia de las emisiones de arco. La utilización de las emisiones de arco para el control y corrección de calidad han sido aplicadas en su mayoría al proceso TIG sin adición de material (Tungsten Inert Gas) debido a que la fusión de los materiales a ser unidos se produce solo por la transferencia de calor y no de masa y esto hace que los parámetros de soldadura presenten un elevado nivel de estabilidad facilitando la detección de perturbaciones ajenas a la transferencia de calor. Esto también facilita el desarrollo de algoritmos recursivos de bajo costo computacional. Sin embargo en el proceso MAG-S la fusión de materiales se produce por la transferencia de calor y de masa lo que hace que la dinámica de transferencia sea caótica. En el proceso de soldadura MAG-S, la transferencia metálica se produce durante los cortocircuitos. Esta complejidad en la transferencia de calor y metal, implica que los algoritmos de control y corrección de interferencias presenten elevadas tasas de muestreo así como elevados costos computacionales, razón por la cual pesquisas en esta área son reducidas y siendo el proceso MAG-S ampliamente utilizado, su entendimiento y aplicabilidad sería de gran impacto.

\section{Procedimiento Experimental}

Se realizaron experimentos de soldadura en posición plana sobre los cuerpos de prueba siguiendo una trayectoria rectilínea de $180 \mathrm{~mm}$ como se muestran en la Figura 2. En cada conjunto de experimentos se indujo interferencia sobre la región de perturbación, consistentes en grasa y ausencia de gas como se muestra en las Figuras $3 \mathrm{a}$ y $3 \mathrm{~b}$ respectivamente. Los ensayos fueron realizados con los parámetros mostrados en la Tabla 1 y se emplearon como materiales, alambre electrodo AWS A5.18 ER70S-6 de $1 \mathrm{~mm}$ de diámetro, chapas de acero AISI 1020 de $400 \mathrm{~mm}$ x $50 \mathrm{~mm}$ x $6.50 \mathrm{~mm}$ y el gas de protección usado fue la mezcla de argón y anhídrido carbónico M21 (ATAL 5A/Ar 82\% $+\mathrm{CO} 218 \%$ ). 


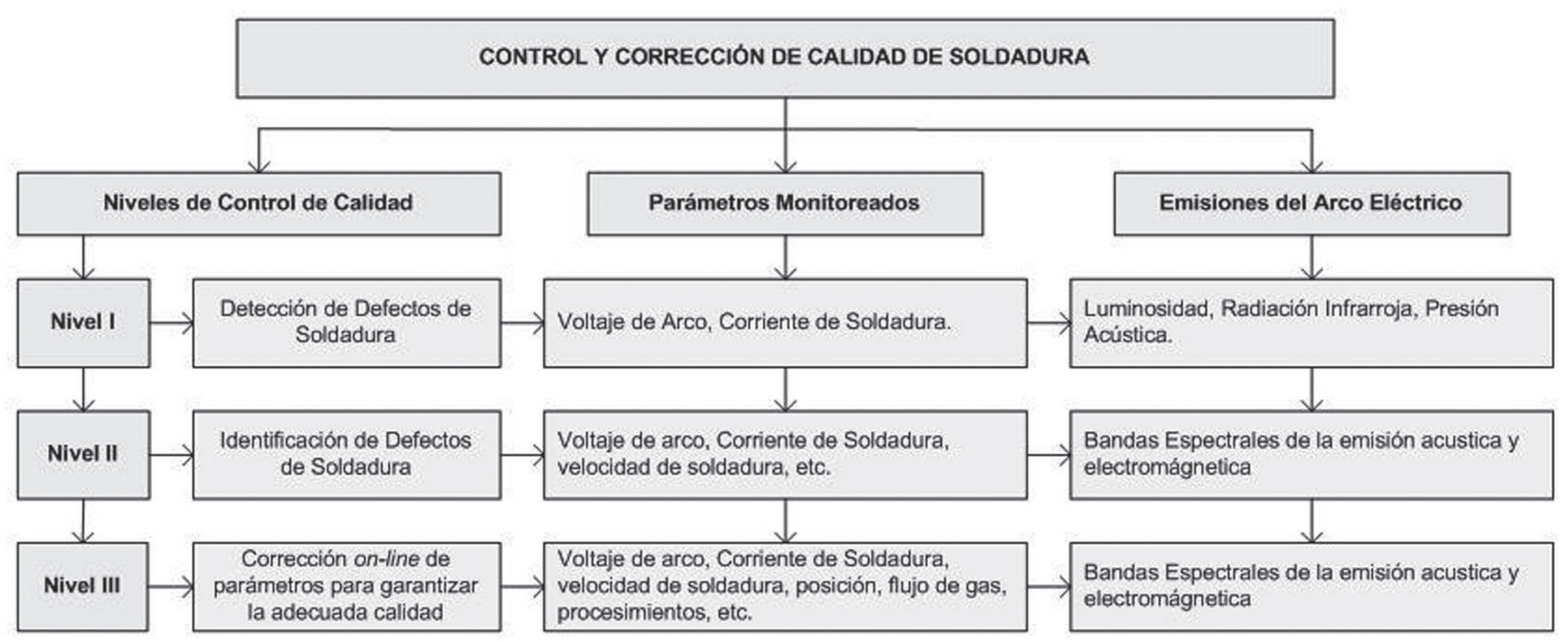

Figura 1. Sistema de Control e Corrección de Calidad on-line.

Tabla 1. Parámetros de Soldadura.

\begin{tabular}{c|c|c|c|c}
\hline $\begin{array}{c}\text { Tensión de arco } \\
(\mathrm{V})\end{array}$ & $\begin{array}{c}\text { Velocidad de } \\
\text { alimentación del } \\
\text { electrodo }(\mathrm{m} / \mathrm{min})\end{array}$ & $\begin{array}{c}\text { Velocidad desplazamiento } \\
\text { de la antorcha de soldadura } \\
(\mathrm{mm} / \mathrm{s})\end{array}$ & $\begin{array}{c}\text { Distancia entre el contacto } \\
\text { del electrodo y el cuerpo } \\
\text { de ensayo (mm) }\end{array}$ & Flujo de Gas (l/min) \\
\hline $20 \pm 0,1$ & $6 \pm 0,1$ & $10 \pm 1,0$ & $12 \pm 1,0$ & $15 \pm 1,0$ \\
\hline
\end{tabular}

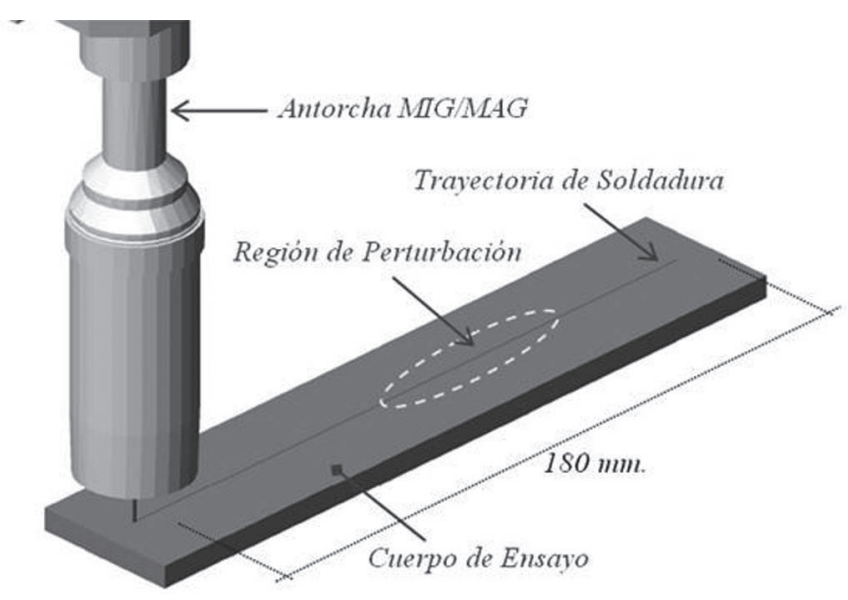

Figura 2. Posición de Soldadura y Localización de

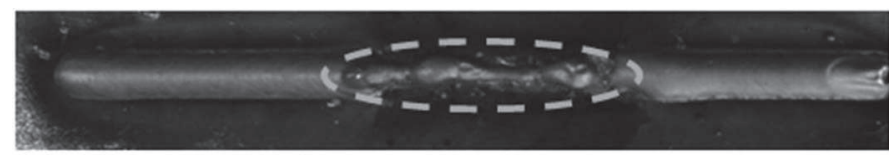

(a)

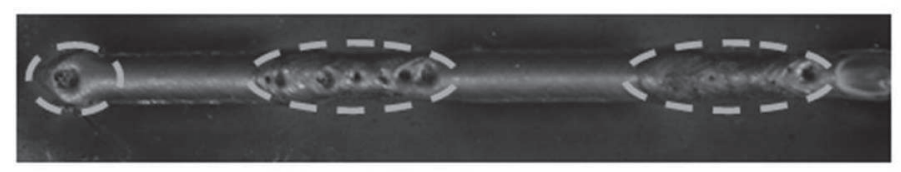

(b)

Figura 3. Experimentos de soldadura con perturbaciones inducidas
Los experimentos fueron realizados utilizando una fuente de energía universal configurada en para el proceso MAG; se adquirieron señales de tensión de arco, corriente de soldadura, presión acústica, intermitencia luminosa y radiación infrarroja; para la medición de la tensión de arco se utilizó un sistema divisor de tensión, un sensor de efecto hall para la medición de la corriente de soldadura, un decibelímetro B\&K - Type 2250 para la medición de las emisiones acústicas (sensor 1), un fotodiodo monolítico con amplificador embarcado (sensor 2) y el pirómetro TL-S-25 Calex (sensor 3) para la medición de las emisiones electromagnéticas en determinadas bandas. Todas las señales nombradas anteriormente fueron muestreadas en simultaneo a una frecuencia de $20 \mathrm{kHz}$ utilizando la tarjeta de adquisición de datos PCI Eagle 703S que es controlado por el software de instrumentación virtual LabVIEW 10. También fue utilizado un sistema de fijación de la antorcha de soldadura en conjunto con una mesa lineal donde se fijó el cuerpo de prueba cuya velocidad avance fue controlada por el computador a través del puerto serial RS 232. Las operaciones de inicio y fin de los experimentos que fueron también controladas desde el computador a través de puertos digitales de la placa de adquisición de datos. En la Figura 4 se muestra la distribución de los equipos utilizados. 


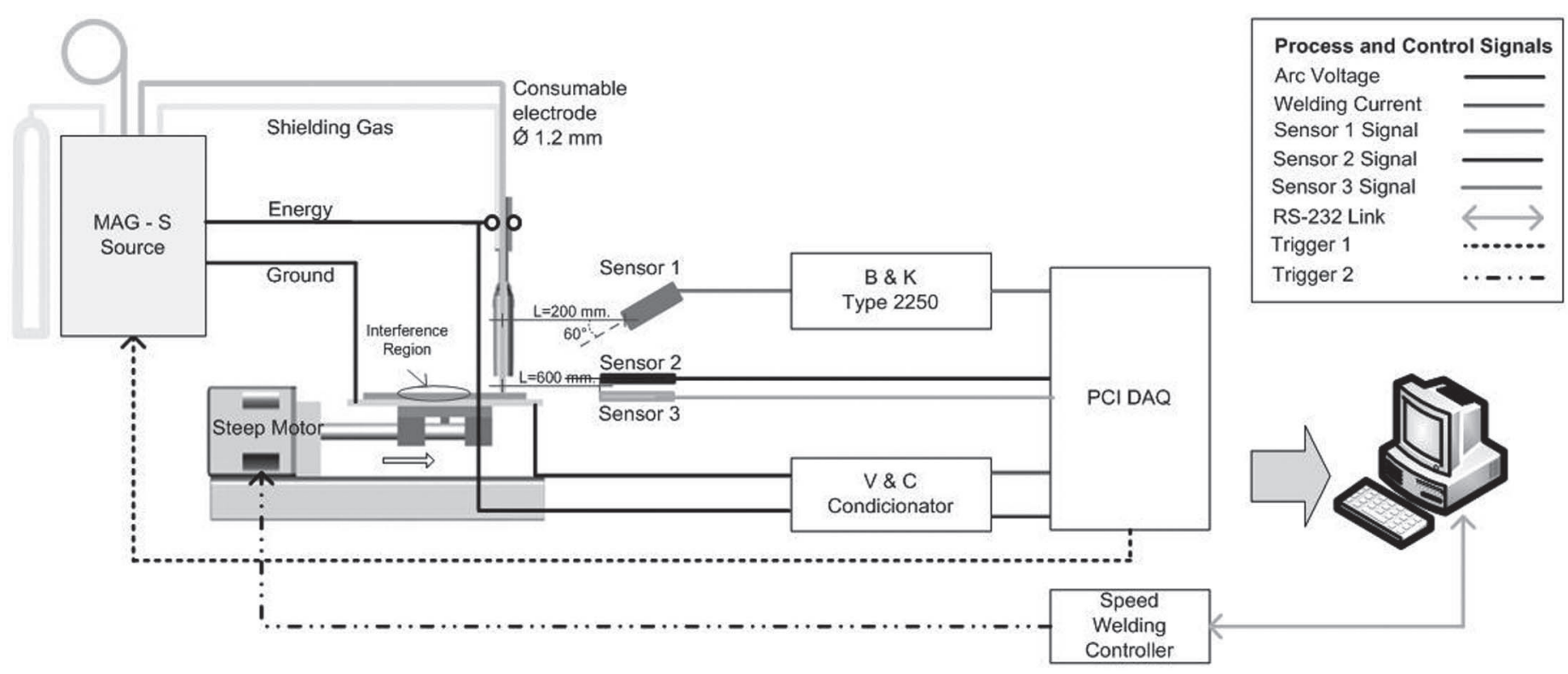

Figura 4. Hardware y Equipos usados en la Experimentación.

\section{Resultados y Discusión}

\subsection{Emisiones Acústicas y Electromagnéticas}

El establecimiento del arco eléctrico es posible debido al flujo de electrones que son acelerados por la diferencia de potencial entre el electrodo consumible y la poza de fusión. En el proceso MAG-S, el arco eléctrico es protegido por un gas activo (mezcla de argón y anhídrido carbónico) y esto facilita la formación del plasma que genera emisiones de naturaleza electromagnética que se manifiestan en los espectros ultravioleta, luz visible e infrarroja. Junto con estas emisiones electromagnéticas, también se producen emisiones acústicas que son de naturaleza mecánica. En el caso del proceso MAG-S, el arco eléctrico presenta una dinámica que está constituida por una secuencia de igniciones y extinciones. Durante la ignición del arco, se produce la transferencia de calor desde la punta del electrodo hasta la poza de fusión. El avance del electrodo hace que se produzca el contacto entre el electrodo y la poza de fusión, momento en el cual se produce el cortocircuito que consecuentemente extingue el arco, cae súbitamente el voltaje del arco y se incrementa la corriente de soldadura. En ese instante se produce la transferencia de metal hasta producirse una nueva ignición del arco (el voltaje de arco se incrementa súbitamente y cae la corriente de soldadura) para continuar con el próximo ciclo de transferencia, como se puede apreciar en las Figuras $5 \mathrm{a}$ y $5 \mathrm{~b}$.

La secuencia de igniciones y extinciones de arco se manifiesta como una secuencia de pulsos acústicos que representa indirectamente el comportamiento del voltaje de arco y corriente de soldadura. Un impulso de menor amplitud se produce cuando una extinción de arco así como un impulso de gran amplitud se produce cuando una ignición de arco ocurre (ver Figura 5 c). Cabe resaltar que es posible notar el retardo entre las señales acústicas y electicas que se deben a su velocidad de propagación en el aire, pero que mientras éstas no superen los $400 \mathrm{~ms}$, no se verán comprometidas con la adecuada representación de la dinámica del arco eléctrico [11, 12]. Investigaciones en monitoreo acústico mostraron que existe una relación entre el sonido producido $\mathrm{S}(\mathrm{t})$ y la potencia del $\operatorname{arco} \mathrm{P}(\mathrm{t})=\mathrm{V}(\mathrm{t}) \mathrm{I}(\mathrm{t})$ que es expresada mediante la ecuación 1.

$S_{c}(t)=K\left[\frac{d(P(t))}{d t}\right]$

$\mathrm{K}=\alpha(\gamma-1) / \mathrm{c}^{2}$

Donde: $\mathrm{S}_{\mathrm{c}}(\mathrm{t})$ es la señal de sonido calculada, $\mathrm{K}$ e el factor de proporcionalidad, $\alpha$ es el factor geométrico, $\gamma$ el coeficiente de expansión adiabática del aire y c la velocidad del sonido en el arco.

En la Figura 5d se puede apreciar el comportamiento de la emisión electromagnética en forma de intermitencia luminosa y puede notarse que cuando se produce una extinción de arco (corto circuito), el nivel de luminosidad llega a ser prácticamente nulo. La respuesta de este sensor no presenta un retardo pronunciado como en el caso del sensor acústico. La intermitencia luminosa fue obtenida a partir del filtrado óptico de las emisiones electromagnéticas del arco en la banda de 380 - $430 \mathrm{~nm}$ que corresponde a las emisiones ultravioletas.

La Figura 5e muestra el comportamiento de la emisión electromagnética en la banda infrarroja. Puede notarse que esta señal no sigue las variaciones de la dinámica del arco, sin embargo en secciones posteriores de este trabajo se mostrara su factibilidad para monitorear variaciones anómalas en la transferencia metálica. La emisión infrarroja es una emisión electromagnética cuya longitud de onda oscila entre 0.8 a 1.1 $\mu \mathrm{m}$. Su intensidad esta directamente ligada a los parámetros de soldadura pre-establecidos tales como material base, composición de gases, niveles de voltaje y corriente. La 
intensidad electromagnética II esta governada por la ley de Planck que describe la radiancia espectral de ondas electromagnéticas no polarizadas para todas las longitudes de ondas emitidas por cuerpos negros a temperatura absoluta T. Como una función de la frecuencia v, la ley de Planck es escrita como:

$I(v, T)=\frac{2 h v^{3}}{c^{2}} \frac{1}{e^{\left.\frac{h \mathrm{hv}}{\mathrm{kT}}\right]_{-1}}}$

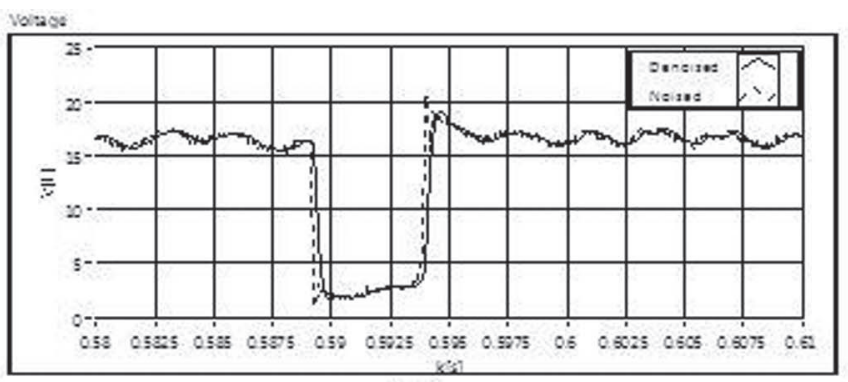

(a)

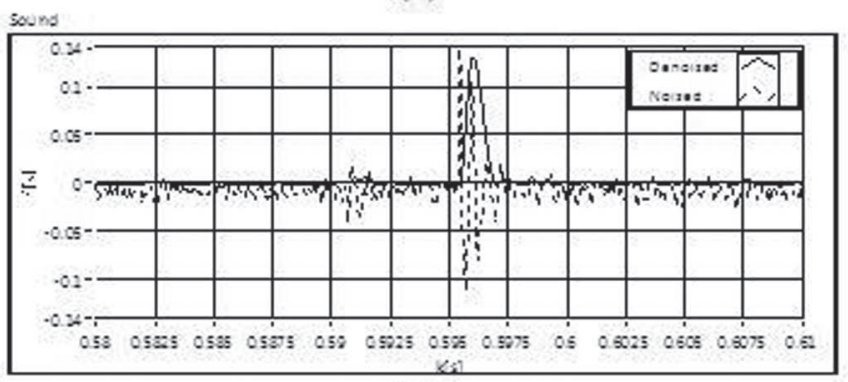

(c)
Donde: I es también denominada radiancia espectral ( J. $\mathrm{m}^{2} \cdot \mathrm{sr}^{-1}$ ), $\mathrm{T}$ temperatura $(\mathrm{K}), \mathrm{v}$ freciencia $(\mathrm{Hz}), \mathrm{h}$ constante de Planck (6.62606896(33) ×10 -34 J.s), c velocidad de la luz $(3.0 \times 108 \mathrm{~m} / \mathrm{s})$ y $\mathrm{k}$ constante de Boltzmann $(\approx 1.3806504 \times$ $10-23 \mathrm{~J} / \mathrm{K})$.



(b)

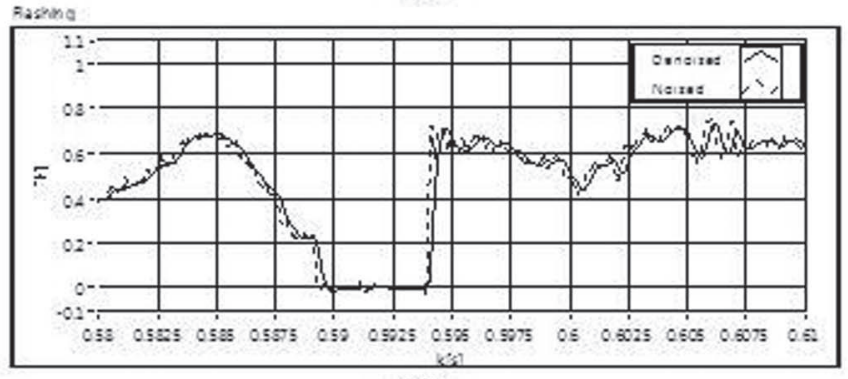

(d)

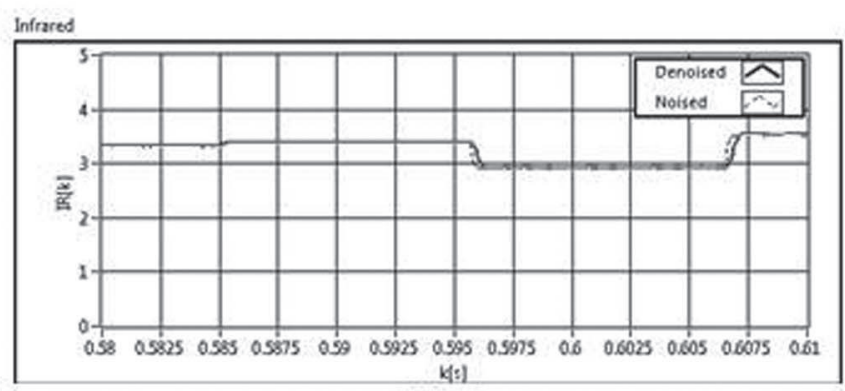

(e)

Figura 5. Señales monitoreadas en el dominio del tiempo

En cada una de las Figuras 5a, 5b, 5c, 5d y 5e se observa las señales en el dominio del tiempo con la presencia de ruido eléctrico así como también la misma señal después de haber sido reducido el nivel de ruido. Para ello se utilizaron filtros digitales pasa bajos Butterworth de segundo orden con una frecuencia de corte de $1 \mathrm{kHz}$. Las Figuras 6a, 6b, 6c, 6d y 6e, muestran el comportamiento de las señales monitoreadas en el dominio de la frecuencia. En estas Figuras puede notarse como el espectro de frecuencias se altera después de reducir el nivel ruido.

\subsection{Relación entre Parámetros de Soldadura y Emisiones de Arco}

La transferencia metálica en el proceso MAG-S se produce en su mayor parte durante los periodos de corto circuito (Ver
Figuras 5a y 5b). Como puede notarse en las Figuras 5c y 5d estas oscilaciones también son reflejadas en el comportamiento de las emisiones de arco. Las variaciones de tensión y corriente son indicadores de variación de la potencia del arco eléctrico. Lo que se refleja en el comportamiento de las emisiones de arco.

Diversos parámetros fueron extraídos a partir de las emisiones de arco. A partir de las emisiones acústicas y las emisiones electromagnéticas en la banda ultravioleta (sensor 1 y 2) se obtuvieron la frecuencia de cortocircuitos. En la Figura 7 se puede observar oscilogramas de la potencia del arco eléctrico y el comportamiento de la frecuencia de cortocircuitos medidos a partir de las emisiones acústicas y luminosas. Nótese que a medida que se incrementa la potencia la frecuencia de cortocircuitos disminuye debido a que el modo de transferencia (cortocircuito) se acerca al modo globular o al modo de 


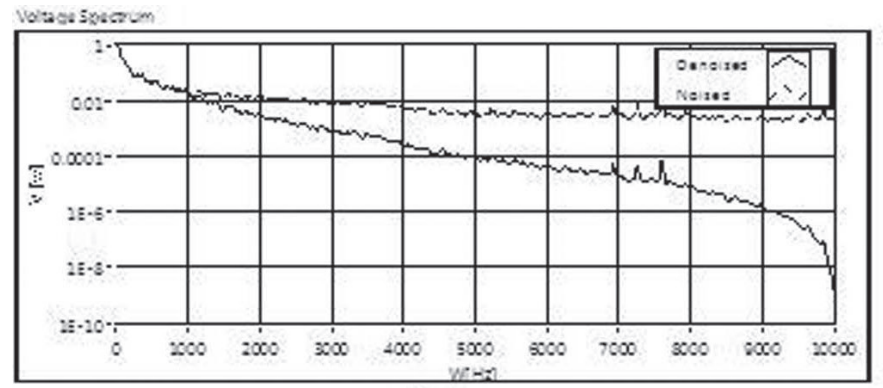

(a)

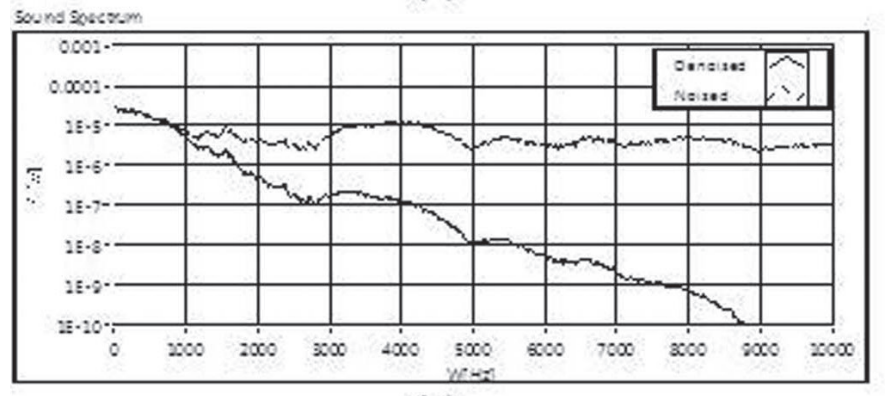

(c)

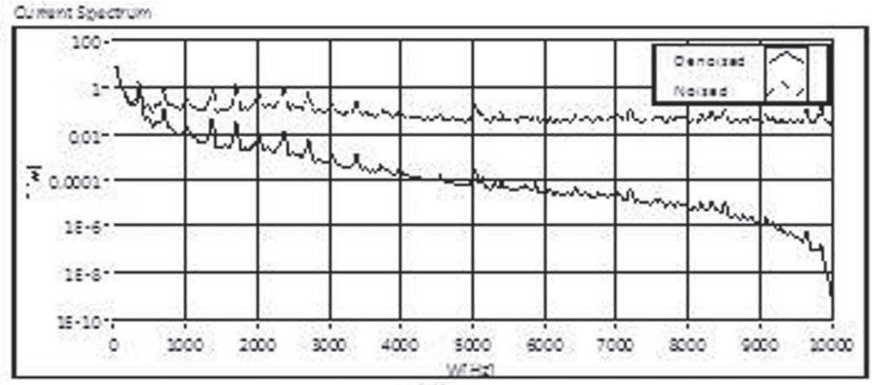

(b)

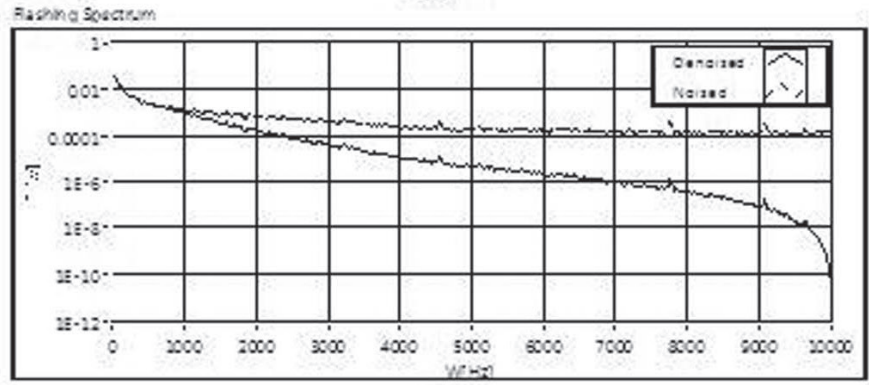

(d)

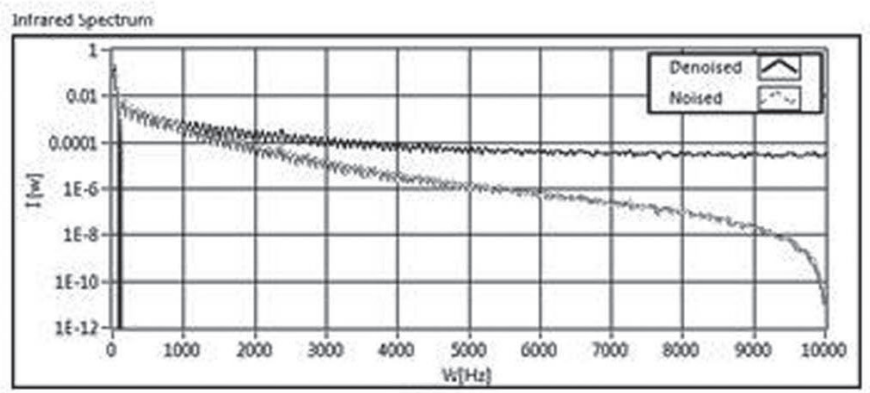

(e)

Figura 6. Señales monitoreadas en el dominio de la frecuencia

pulverización axial donde la presencia de cortocircuitos es prácticamente nula. Nótese también que las mediciones de la frecuencia de cortocircuitos a partir de las emisiones luminosas presentan más estabilidad que las mediciones hechas a partir de las emisiones acústicas. Además de ello puede notarse también que el efecto aerotransportado del sonido se manifiesta como un retardo en la medición de la frecuencia de cortocircuitos.

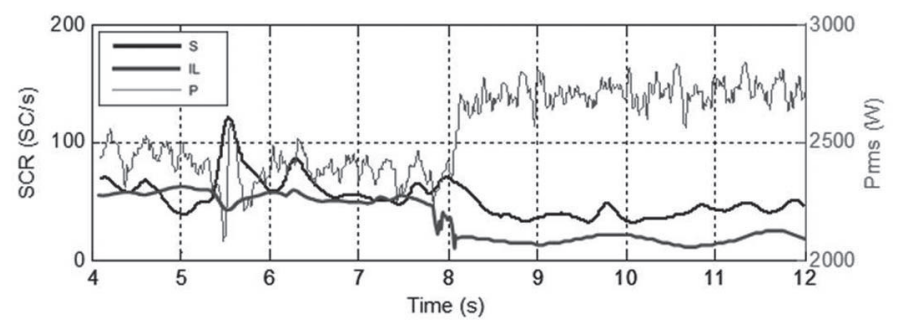

Figura 7. Emisión acústica y electromagnética (ultravioleta) y su relación con la potencia de arco

Las mudanzas de potencia de arco producen mudanzas en la temperatura tanto de la poza de fusión como del arco. En la Figura 8 pueden observarse los oscilogramas de la potencia del arco eléctrico y la respuesta del pirómetro (sensor 3) que mide las emisiones infrarrojas. Nótese que a pesar que la respuesta de este sensor no consigue representar la dinámica de la transferencia de masa en el proceso MAG-S, éste puede representar el comportamiento de la potencia del arco. En comparación con el sensor de emisiones ultravioletas, este sensor es sensible frente a las variaciones de potencia.

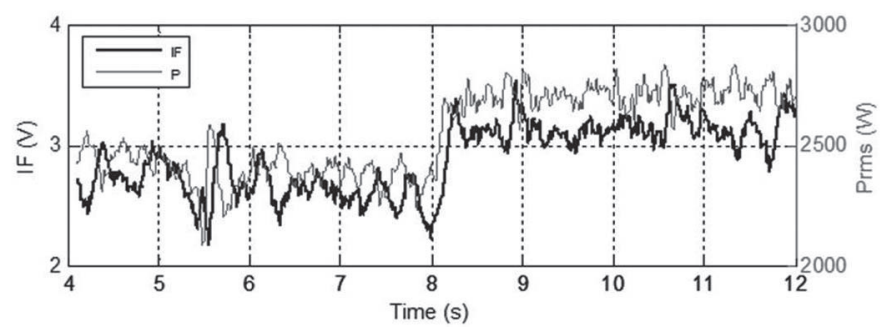

Figura 8. Emisión electromagnética (infrarrojo) y su relación con la potencia de arco

\subsection{Detección de Perturbaciones}

La estabilidad en el proceso MAG-S está directamente relacionada a las oscilaciones de la poza de fusión y alcanza un 
máximo umbral cuando el ratio de corto circuitos es máximo, la desviación estándar del ratio de corto circuitos es mínima, el volumen de masa transferida por cortocircuito mínimo y el nivel de salpicadura son mínimos, [6-9]. Se espera que durante una soldadura estos parámetros y/o características de la transferencia metálica se mantenga inalterables y mudanzas súbitas indicarán la falta de estabilidad pudiendo esto estar relacionado con la presencia de alguna perturbación y/o defecto. Para ello, a partir de las emisiones acústicas y electromagnéticas se obtuvo la frecuencia de cortocircuitos y se monitoreo la respuesta de estas señales frente a la presencia de perturbaciones inducidas. Las Figuras 9 y 10 (a), (b), (c) y (d) muestran la tensión y corriente RMS del arco, frecuencia de cortocircuitos a partir de emisiones acústicas, frecuencia de de cortocircuitos a partir de emisiones ultravioleta y emisión infrarroja que fueron monitoreados para experimentos de soldadura sometido a las interferencias por presencia de grasa y ausencia de gas de protección respectivamente. La presencia de grasa en la poza de fusión alterara dramáticamente tanto en los gradientes de temperatura así como también en la geometría final del cordón de soldadura. La ausencia de gas de protección reduce bruscamente la ionización del aire en torno del arco eléctrico y junto con la relativa baja tensión de arco (en torno de $20 \mathrm{~V}$ ) conlleva a la reducción de la taza de transferencia de calor y por tanto a la presencia de porosidades en el cordón de soldadura.

Puede apreciarse que para el primer experimento, la tensión y corriente de arco manifiestan variaciones no pronunciadas

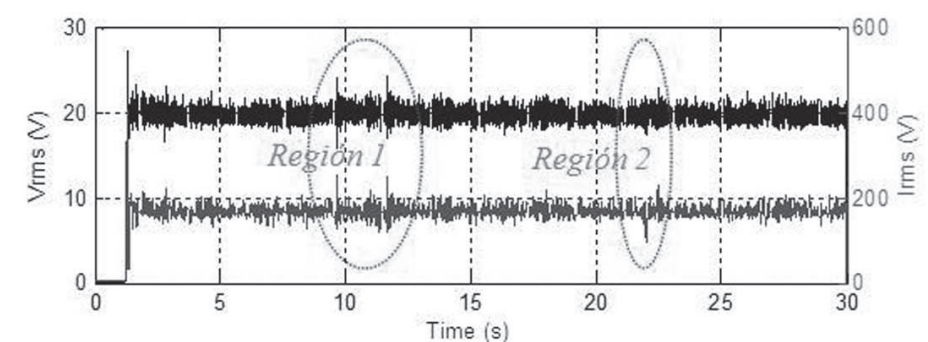

(a)

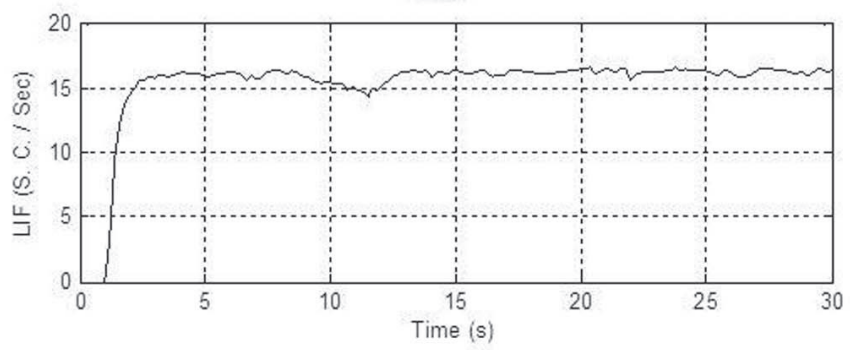

(c) frente a la presencia de grasa. No obstante ello la frecuencia de de cortocircuitos medido a partir de las emisiones acústicas y ultravioletas, presentan variaciones relativamente pronunciadas. Sin embargo la señal de radiación infrarroja presento un súbito incremento en su magnitud para la primera región con presencia de grasa, hecho que refleja la presencia de una perturbación. Nótese que la frecuencia de cortocircuitos medida a partir de las emisiones acústicas, presta un elevado nivel de rizado en comparación con la medición hecha a partir de las emisiones ultravioleta. En la Figuras 11 y 12 se muestran los espectrogramas de las emisiones acústicas para los experimentos antes mencionados. Puede notarse que existen diversas componentes de frecuencia donde la frecuencia dominante corresponde aproximadamente a $2 \mathrm{kHz}$. El espectro de frecuencias de las emisiones acústicas presenta un conjunto de componentes relacionadas al comportamiento del plasma así como también al ruido ambiental que normalmente son elevados en ambientes de trabajos en soldadura. Sin embargo lo que se monitorea son las explosiones producidas por los cortocircuitos y estas se presentan a bajas frecuencias (80 cortocircuitos por segundo). En presencia de perturbaciones puede observarse que se presentan intermitencias tanto en el dominio del tiempo como de la frecuencia. Dado que la presencia de perturbaciones produce alteraciones súbitas, caóticas y temporales, no fue posible identificar frecuencias características que representen la presencia de perturbaciones.

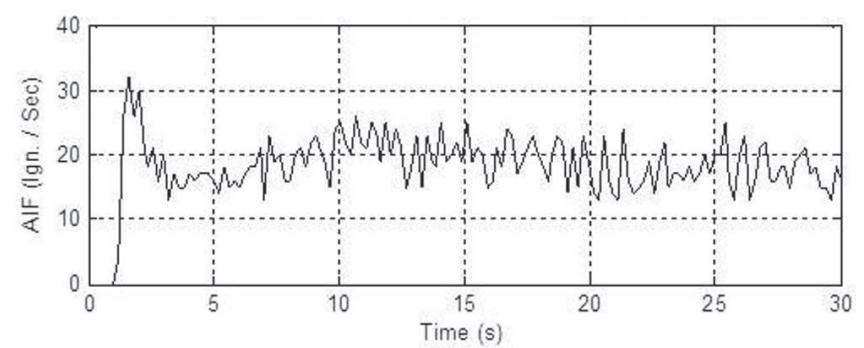

(b)

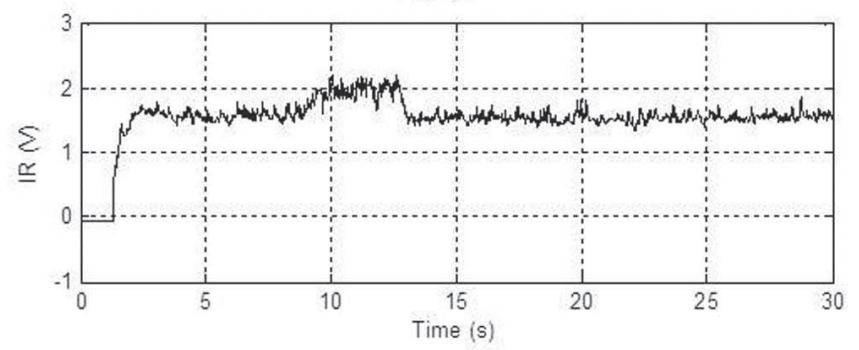

(d)

Figura 9. Experimento con perturbación 1 


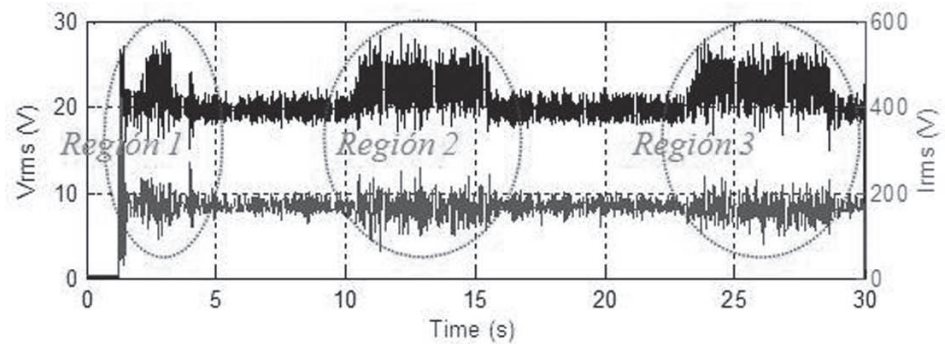

(a)

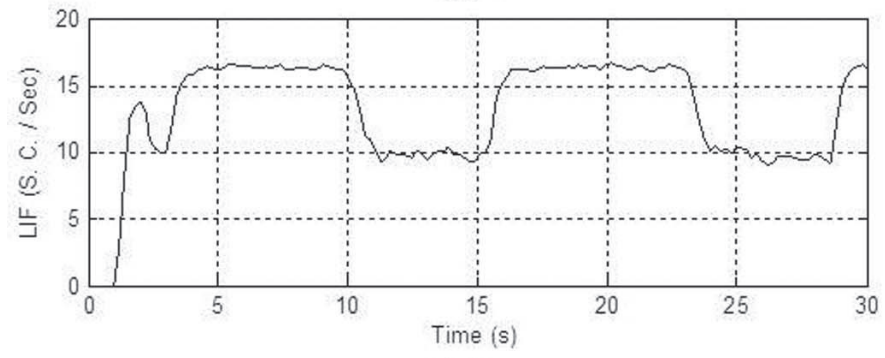

(c)



(b)

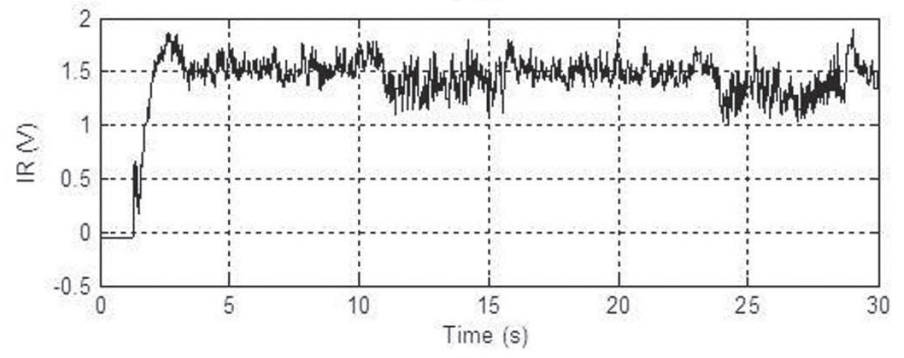

(d)

Figura 10. Experimento con perturbación 2

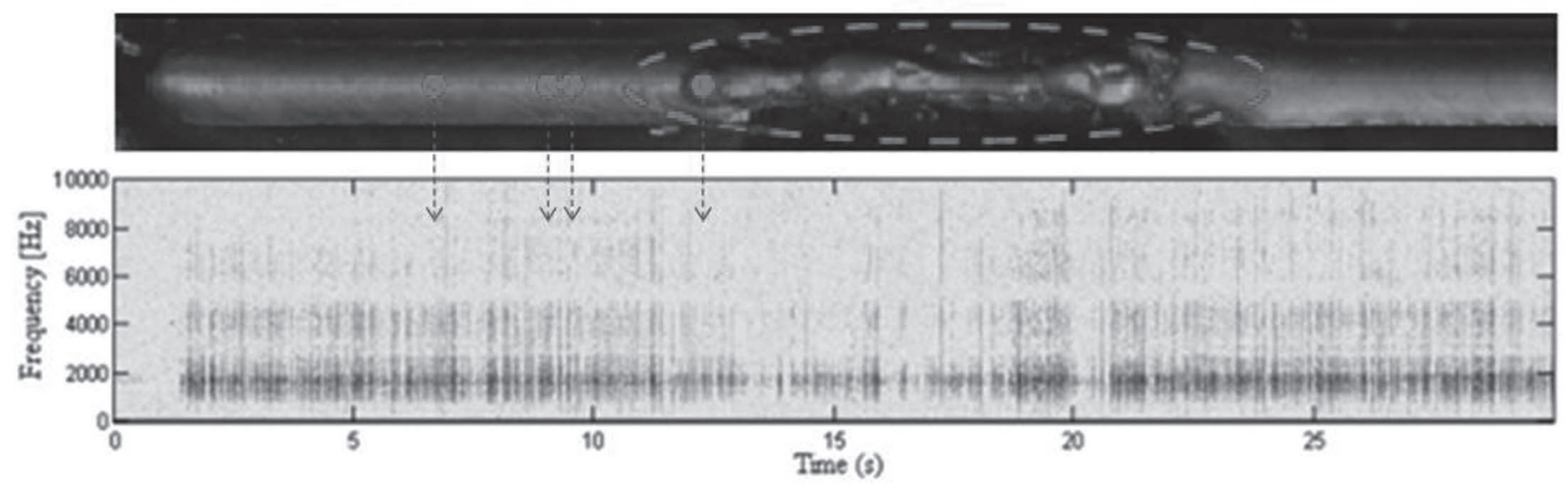

Figura 11. Respuesta de las emisiones de arco frente a la perturbación simulada 1

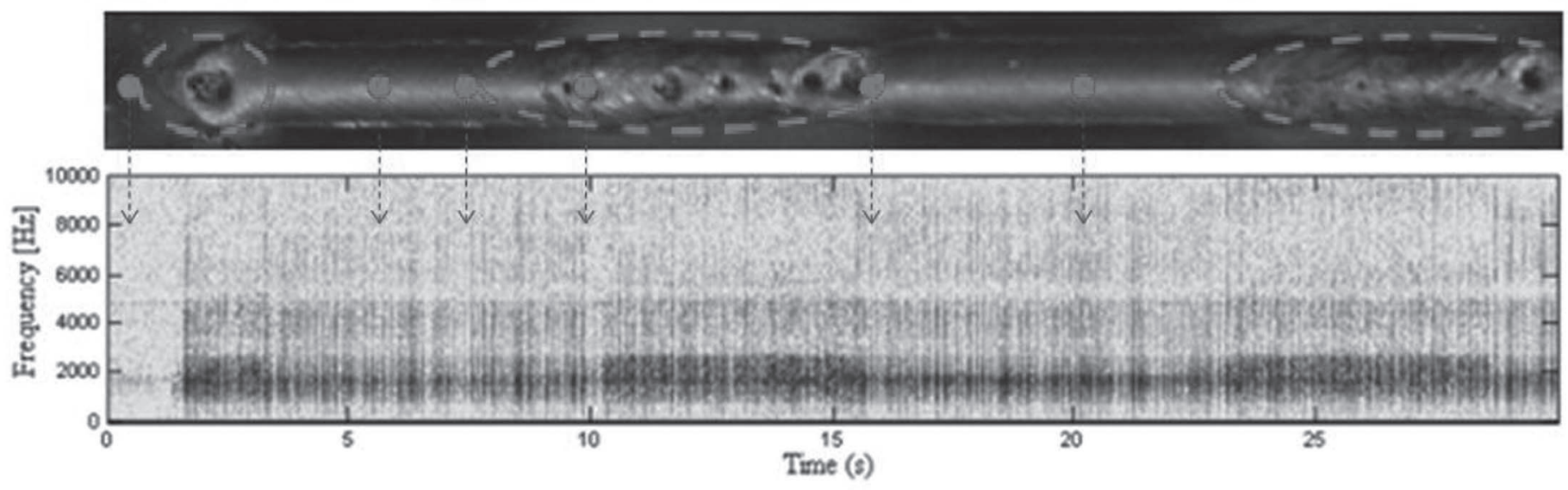

Figura 12. Respuesta de las emisiones de arco frente a la perturbación simulada 2 
En la Figura 12 puede notarse que para el segundo experimento tanto la tensión como la corriente varían expresivamente frente a la perturbación por ausencia de gas de protección. Estas variaciones también se reflejaron en las emisiones de arco como un incremento en la frecuencia de cortocircuitos monitoreado por las emisiones acústicas y un decremento en la frecuencia de cortocircuitos monitoreado por las emisiones ultravioletas. Esto de alguna manera inicialmente resulta contradictorio ya que se trata del mismo proceso monitoreado por diferentes sensores. Las emisiones acústicas muestran que el numero de cortocircuitos se ha incrementado, sin embargo, estas emisiones acústicas proviene de la oscilación de la poza de fusión (que se ha visto altamente contaminada por la presencia de oxigeno) y no por las explosiones de los cortocircuitos. Las emisiones ultravioleta muestran que el número de cortocircuitos, es decir las intermitencias luminosas se han reducido. La ausencia de gas de protección también se manifestó con la reducción del nivel de emisión infrarroja para las tres regiones de perturbación.

\section{Conclusiones}

Las señales de tensión de arco, corriente de soldadura, emisiones acústica, ultravioleta e infrarroja fueron evaluadas para el entendimiento de la relación entre parámetros de soldadura y emisiones del arco eléctrico para el proceso de soldadura MAG-S. Frente a la presencia de disturbios simulados, cada emisión del arco presentó comportamientos caóticos que permitieron monitorear inestabilidades y/o perturbaciones en la trayectoria de soldadura. A partir de la emisión acústica y ultravioleta se midió la frecuencia de cortocircuitos, en tanto que la emisión infrarroja fue usada como parámetro directo de monitoreo de cambios súbitos relacionados a la temperatura de la poza de fusión. La respuesta presentada por las emisiones del arco frente a disturbios inducidos en la trayectoria de soldadura, mostró la posibilidad de utilizar la emisiones para el monitoreo y detección de perturbaciones en el control de calidad así como también la posibilidad de utilizarlas como variables de realimentación en sistemas de control de transferencia de masa para el proceso MAG-S.

\section{Agradecimientos}

Los autores agradecen por el soporte de éste proyecto a La Universidad de Brasilia y al CNPq (Consejo Nacional de Desenvolvimiento Científico y Tecnológico).

\section{Referencias Bibliográficas}

[1] KRALJ, V., Biocybernetic investigations of hand movements of human operator in hand welding, IIW/IIS Doc. 212-140-68, 1968.

[2] TAM, J.; HUISSOON, J. Developing Psycho-Acoustic Experiments in Gas Metal Arc Welding. In; Proceedings of the IEEE, International Conference on Mechatronics \& Automation, Niagara Falls, Canada. 2005, p. 1112-1117.

[3] MIRAPEIX, J.; COBO, A.; GONZÁLEZ, D.A.; LÓPEZHIGUERA, J.M. Plasma spectroscopy analysis technique based on optimization algorithms and spectral synthesis for arcwelding quality assurance. In: Optics Express. 2007, Vol. 5, no. 4, p. 1884-1889.

[4] CAYO, H.C.; ALFARO, S.C.A. Non-Intrusive GMA Welding Process Quality Monitoring System Using Acoustic Sensing, Sensors, 9, 2009 p. 7150-7166.

[5] SIMPSON, S.W.; HUGHES, P. Prospects for Fault identification and control in welding using signature images, $\mathrm{N}$. S. W. 2006, School of electrical and Information Engineering, University of Sydney, Australia

[6] BINGUL, Z.; COOK, G.E. Dynamic Modeling of GMAW Process. In: Proceeding of the 1999 IEEE International Conference on Robotics \& Automation, Detroit, MI, USA. 1999. p. 3059-3064.

[7] WU, C.S.; POLTE, T.; REHFELDT, D. A Fuzzy Logic System for Process Monitoring and Quality Evaluation in GMAW. In: Supplement to the Welding Journal, February 2001. p. 33-38.

[8] HERMANS, M.J.; DEN, O.G. Process Behavior and Stability in Short Circuit Gas Metal Arc Welding. In: Welding Research Supplement, April 1999, p. 137-141.

[9] ADOLFSSON, S.; BAHRAMI, A.; BOLMSJÖ, G.; CLAESSON, I. Quality Monitoring in Robotized Spray GMA Welding. In: Inst. J. for the Joining of Materials 10 (1/2), 1998. [10] GRAD, L.; GRUM, J.; POLAJNAR, I.; SLABE, J.M. Feasibility study of acoustic signals for on-line monitoring in short circuit gas metal arc welding. In: International Journal of Machine Tools and Manufacture Volume 44, Issue 5, April 2004, p. 555-561.

[11] DROUET, M.G.; NADEAU, F. Pressure Waves due to Arcing Faults in a Substation. In: IEEE Transactions on Power Apparatus and Systems, 1979, 5, p. 98.

[12] DROUET, M.G.; NADEAU, F. Acoustic measurement of the arc voltage applicable to arc welding and arc furnaces. In: J. Phys. E: Sci. Instrum., 1982, 15, p. 268-269. 